\title{
Study on clinical effects of pedicle screw combined with immobilized implantation bone by wirerope and hallow compression screw immobilization in treating spondylolysis of lumbar vertebra of multiple segments of adults
}

\author{
Dongdong Zhao*1, Feng $\mathrm{Li}^{1}$, Yao $\mathrm{Wu}^{1}$, Xiaoyan Zhang ${ }^{2}$ \\ ${ }^{1}$ The First Section of Orthopedics Department of Huhhot First Hospital, Huhhot, Inner Mongolia, China \\ ${ }^{2}$ Department of Orthopedics, Baogang Hospital, Baotou, Inner Mongolia, China
}

Received: November 5, 2019

Accepted: February 30, 2020

Online Published: April 22, 2020

DOI: $10.5430 /$ dcc.v6n3p19

URL: https://doi.org/10.5430/dcc.v6n3p19

\begin{abstract}
Objective: To observe clinical effects of pedicle screw fixation combined with cable wires and bone graft and cannulated compression screws on adult multi-segment lumbar spondylolysis.

Methods: 70 cases of patients with multi-segment lumbar spondylolysis were selected in our hospital. According to different surgical schemes, these patients were divided into the observation group (35 cases) and the control group (35 cases). The observation group received pedicle screw fixation combined with cable wires and bone graft and the control group received cannulated compression screw fixation. Macnab criteria were adopted to implement a therapeutic evaluation of two groups of patients to make an observation and comparison of the excellent and good rate of surgery and a series of indicators including perioperative clinical effects, intraoperative blood loss, duration of surgery, hospital length of stay (HLOS), visual analogue scale (VAS), Oswestry disability index and Japanese Orthopaedic Association (JOA) score.

Results: The excellent and good rate of the observation group was $97.14 \%$, and that of the control group was $82.86 \%$, the difference between two groups was statistically significant $\left(\chi^{2}=6.248, p=.012\right)$. The differences in intraoperative blood loss, duration of surgery and HLOS between two groups were statistically significant $(t=-4.55, t=-4.55, t=-4.55 ; p<.05)$. Oswestry index, VAS score and JOA score of the observation group were $(2.4 \pm 0.9),(28.5 \pm 6.4)$ and $(27.1 \pm 3.1)$ respectively, and these of the control group were $(3.5 \pm 1.2),(37.1 \pm 7.8)$ and $(21.3 \pm 2.7)$ respectively, the differences between two groups were statistically significant $(t=4.338, t=5.043, t=8.347, p<.05)$.

Conclusions: Pedicle screw combined with immobilized implantation bone by wirerope has an excellent clinical effect on the treatment of adult multi-segment lumbar spondylolysis, and it has a series of advantages such as fast postoperative recovery, small surgical trauma and so on. In addition, this technique can also restore the stability of spinal segments and relieve pains to a greater degree.
\end{abstract}

Key Words: Pedicle screw, Cable wires and bone graft, Cannulated compression screw fixation, Adult multi-segment lumbar spondylolysis

\footnotetext{
*Correspondence: Dongdong Zhao; Email: nmgbgyyyy @ 163.com; Address: The First Section of Orthopedics Department of Huhhot First Hospital, Huhhot, Inner Mongolia 010030, China.
} 


\section{INTRODUCTION}

Lumbar spondylolysis is a commonly-seen pathological change of lumbar vertebra clinically, with the main characteristic of bone nonunion in lumbar pedicle isthmus. ${ }^{[1]}$ Multi-segment lumbar spondylolysis is a commonly-seen type of lumbar spondylolysis. After the occurrence, it easily causes the instability of lumbar vertebra and intractable low-back pain and so on, even leads to some clinical manifestations such as intermittent claudication and double lower limb numbness, severely affecting patients' life quality. ${ }^{[2,3]}$ In this research, pedicle screw fixation combined with cable wires and bone graft was performed in the treatment of adult multi-segment lumbar spondylolysis.

\section{DATA AND METHODS}

\subsection{General information}

70 cases of adult patients with multi-segment lumbar spondylolysis were chosen from Huhhot First Hospital during January of 2012 to January of 2015. According to different surgical schemes, these patients were divided into the observation group ( 35 cases) and the control group (35 cases). In the observation group, there were 22 cases of male patients and 13 cases of female patients, aged from 24 to 52, mean age of $(40.5 \pm 5.7)$, and they received pedicle screw fixation combined with cable wires and bone graft. In the control group, there were 23 cases of male patients and 12 cases of female patients, aged from 23 to 53, mean age of ( $40.8 \pm 5.4)$, and they received cannulated compression screw fixation. It was comparable in the clinical baseline information between two groups of patients due to no statistical difference.

\subsection{Inclusion and exclusion criteria}

(1) Inclusion criteria: i. Patients had chief complaints of lumbodynia and skelalgia (mostly lumbodynia); ii. According to 3D CT and X-ray examination (antero-posterior and bilateral oblique roentgenography) of lumbar vertebra, it was clearly diagnosed as multi-segment lumber spondylolysis (bilateral). (2) Exclusion criteria: i. Patients with other severe internal medicine diseases and intolerance of operation in the preoperative assessment; ii. Patients with unilateral spondylolysis; iii. Patients with mental disorder and who cannot cooperate with doctors during treatment.

\subsection{Surgical methods}

\subsubsection{Surgical scheme for the observation group}

The pedicle screw fixation combined with cable wires and bone graft was performed in the method of posterior approach operation. L3 to L5 vertebral laminas, intervertebral facet joints and transverse processes were exposed adequately, and it can be seen through the inspection that L3 to L5 vertebral laminas were floating with the fracture of pedicle isthmus and local formation of pseudarthrosis. In addition, osseous and soft tissue hyperplasia can be obviously seen during surgery. In the process of treatment, it was required to thoroughly remove the hyperplasia tissues of pseudarthrosis and locate the point of pedicle screw insertion. Drilled holes on L3 to L5 bilateral pedicles of vertebral arch with the orthopaedic bone tap, with two pedicle screws inserted respectively. Each pedicle screw was equipped with a piece of titanium wire, which came around the spinous process and then passed through the end of pedicle screw and returned from isthmus to be tightened and fixed with the screw cap. Took bones from posterior superior iliac spine and then placed them into L3 to L5 bilateral isthmus respectively. After surgery, patients needed to follow the doctor's advice to take bed rest and a recovery. Generally, off-bed activities were not allowed only until 3 months passed (depending on the state of recovery).

\subsubsection{Surgical scheme for the control group}

Cannulated compression screw fixation was applied in the surgery, with continuous epidural anesthesia adopted. Patients' lower limbs were well placed on the special extension device, and the traction and reposition of fracture ends was performed under the observation of $\mathrm{C}$-arm $\mathrm{X}$-ray equipment.

Depending on the state of recovery, after surgery, both groups of patients needed to take bed rest for about 3 months and receive conventional anti-infective treatment.

\subsection{Indicator observation}

\subsubsection{Post-operative therapeutic effect}

Macnab criteria were adopted to evaluate the excellent and good rate in each group: (1) Excellent: patients with no obvious pain and limitation of motion after surgery, can participate in the regular work and activities; (2 )Good: patients with occasional non-neuropathic pain, but the main symptoms had been relieved, can participate in the adjusted work; (3) Satisfactory: patients with a considerably improved function, but stayed in a disabled and/or unemployed state; (4) Bad: Patients were diagnosed as injured nerve rootlet after surgery, with recurrent clinical symptoms.

\subsubsection{Perioperative indicators}

During the perioperative process, visual analogue scale (VAS), Oswestry disability index and Japanese Orthopaedic Association (JOA) score were applied to the evaluation on the level of pain, the state of recovery and joint dysfunction in two groups of patients: (1) VAS has a special scale, one end of which was set as 10 scores and the other end was 0 score, 10 was for the most severe pain and 0 was for no pain, i.e., it was indicated that the higher score meant the more severe pain; (2) Oswestry disability index contained 10 items, 
such as social activities, the level of pain and so on, which were represented by $0-5$ scores. The higher score indicated the more severe joint dysfunction; (3) JOA score consisted of 3 items (motor function, sensory function and bladder function). Motor function could be divided into upper limb function and lower limb function, which were rated by 0-4 scores; sensory function could be divided into upper limb function and lower limb function, which were rated by 0-2 scores; and bladder function was rated by $0-3$ scores. The total score could be calculated by adding up the score of each item. Higher score indicated the better state of recovery after surgery.

\subsubsection{The evaluation of the perioperative condition}

It was necessary to evaluate intraoperative blood loss, duration of surgery and HLOS.

\subsection{Statistical methods}

SPSS 17.0 statistical software was applied to the treatment and analysis of statistical data. The measurement data fitted to normal distribution, and the comparison between two groups was made by use of $t$-test. The categorical data were represented by $(\bar{x} \pm \mathrm{s})$ and percentage $(\%)$, with $\chi^{2}$ test performed. The difference $p<.05$ was of statistical significance.

\section{Results}

\subsection{The comparison in the therapeutic effect between two groups of patients}

The excellent and good rate of the observation group $(97.14 \%)$ was obviously higher than that of the control group
$(82.86 \%)$. The difference was of statistical significance $\left(\chi^{2}\right.$ $=6.248, p<.05)($ see Table 1$)$.

\subsection{The comparison in the surgical injury and postoper- ative recovery between two groups of patients}

In the observation group, the intraoperative blood loss was $(180.5 \pm 30.6) \mathrm{ml}$, the duration of surgery was (130.5 \pm 12.3$)$ min and HLOS was $(10.2 \pm 1.5) \mathrm{d}$; in the control group, the intraoperative blood loss was $(203.3 \pm 35.5) \mathrm{ml}$, the duration of surgery was $(145.6 \pm 15.3) \mathrm{min}$ and HLOS was $(13.5 \pm$ 2.5) d. It was indicated that the observation group showed less surgical injury and HLOS. The difference was of statistical significance $(t=-4.55, t=-4.55, t=-4.55 ; p<.05)$ (see Table 2).

\subsection{The comparison in perioperative indicators between two groups of patients}

There was no statistically significant difference between two groups of patients in VAS, Oswestry and JOA scores before surgery. However, after surgery, VAS and Oswestry scores in the observation group were obviously lower than those in the control group, and JOA score in the former was significantly higher than that in the latter. The difference was of statistical significance $(p<.05)$ (see Table 3$)$.

\subsection{The comparison in the postoperative complications between two groups of patients}

During the follow-up, all patients had no complications about the rupture, loosening and disconnection of pedicle screws and lamina hooks.

Table 1. The comparison in the clinical therapeutic effect between two groups of patients with multi-segment lumber spondylolysis $[\mathrm{n}(\%)]$

\begin{tabular}{lllllll}
\hline Group & n & Excellent & Good & Satisfactory & Bad & Excellent \& Good Rate (\%) \\
\hline Observation Group & 35 & $28(80.00)$ & $6(17.14)$ & $1(2.86)$ & 0 & 97.14 \\
Control Group & 35 & $20(57.14)$ & $7(20.00)$ & $4(11.43)$ & $2(5.71)$ & 82.86 \\
$\chi^{2}$ & & 4.242 & 0.094 & 0.862 & 0.515 & 6.248 \\
$p$ Value & & .039 & .759 & .353 & .473 & .012 \\
\hline
\end{tabular}

Table 2. The comparison in the surgical injury and postoperative recovery between two groups of patients with multi-segment lumber spondylolysis $(\bar{x} \pm \mathrm{s})$

\begin{tabular}{lllll}
\hline Group & $\mathbf{n}$ & Intraoperative Blood Loss (ml) & Duration of Surgery (min) & HLOS (d) \\
\hline Observation Group & 35 & $180.5 \pm 30.6$ & $130.5 \pm 12.3$ & $10.2 \pm 1.5$ \\
Control Group & 35 & $203.3 \pm 35.5$ & $145.6 \pm 15.3$ & $13.5 \pm 2.5$ \\
$t$ Value & & 3.509 & 4.551 & 6.696 \\
$p$ Value & & .005 & $<.05$ & $<.05$ \\
\hline
\end{tabular}


Table 3. The comparison in the perioperative indicators between two groups of patients with multi-segment lumber spondylolysis (score, $\bar{x} \pm \mathrm{s}$ )

\begin{tabular}{|c|c|c|c|c|c|c|c|}
\hline \multirow{2}{*}{ Group } & \multirow{2}{*}{$\mathbf{n}$} & \multicolumn{2}{|c|}{ VAS } & \multicolumn{2}{|c|}{ Oswestry } & \multicolumn{2}{|c|}{ JOA } \\
\hline & & Preoperative & Postoperative & Preoperative & Postoperative & Preoperative & Postoperative \\
\hline Observation Group & 35 & $6.3 \pm 1.4$ & $2.4 \pm 0.9$ & $51.1 \pm 10.6$ & $28.5 \pm 6.4$ & $18.2 \pm 2.4$ & $27.1 \pm 3.1$ \\
\hline Control Group & 35 & $6.4 \pm 1.1$ & $3.5 \pm 1.2$ & $52.0 \pm 9.8$ & $37.1 \pm 7.8$ & $17.9 \pm 2.7$ & $21.3 \pm 2.7$ \\
\hline$t$ Value & & 0.332 & 4.338 & 0.369 & 5.043 & 0.491 & 8.347 \\
\hline$p$ Value & & .741 & $<.05$ & .713 & $<.05$ & .625 & $<.05$ \\
\hline
\end{tabular}

\section{DISCUSSION}

In the current researches, it is considered that simple pedicle screw fixation may damage patients' nerve roots during surgery or result in the less pressure on the lesion location after fixation because of small contact area of lumber spondylolysis. ${ }^{[4,5]}$ Therefore, simple pedicle screw fixation has an ordinary therapeutic effect, so that it is gradually replaced by other surgical methods. Even though bone grafting based on pedicle screw fixation is of a high fusion rate, patients may prone to the rupture of fixation or the crack of the fusion part since most of load will be passed through pedicle screw system and the fusion area of the posterior column as $80 \%$ of lumbar axial load is passed on through the anterior column. It is more commonly seen in the patients with multi-segment lumber spondylolysis. ${ }^{[6,7]}$

In recent years, pedicle screw fixation and intervertebral fusion surgery has become a preferred choice for the treatment of lumber spondylolysis, with some advantages of solid fixation and high fusion rate. Therefore, it has much superiority in the biomechanical aspect. ${ }^{[8]}$ However, it may result in a long duration of postoperative recovery since it causes a large surgical trauma to the patients. In addition, this surgical method can also cause the loss in the range of motion for the fused vertebral body, accelerating the degeneration of adjacent intervertebral discs. On that account, it is generally not recommended for the patients with multi-segment lumber spondylolysis to receive this type of surgery.

During the process of treating the patients with multisegment lumber spondylolysis, this research adopted tension band wire technique on the basis of pedicle screw fixation. In order to further explore the therapeutic effect of pedicle screw fixation combined with cable wires and bone graft on adult multi-segment lumber spondylolysis, the clinical grouping observation was made to 70 cases of surgical patients in this research. It was indicated that the excellent and good rate in the observation group was obviously higher than that in the control group. As the surgical method mentioned above can furthest reduce the effect of patients' activities on the motion of lumbar vertebra and effectively avoid from stress concentration caused by multi-segment fusion. Therefore, it can significantly reduce the probability of the degeneration of intervertebral discs to improve the therapeutic effect; meanwhile, the perioperative indicators in the observation group prove to be superior to the control group, indicating that it can reduce the surgical injury and reduce the duration of recovery. Moreover, VAS, Oswestry and JOA scores have been applied to the comparison in perioperative pain, joint function, the state of recovery etc. between two groups of patients in this research. The results show that the postoperative pain in the observation group was much more relieved than that in the control group, with joint function and the state of recovery in the former better than those in the latter.

In this research, the adopted surgical method has the following advantages over adult multi-segment lumber spondylolysis: (1) the usage of cable wire can form a tension band on the surface of bone stump; (2) cable wires are thin and have a good fatigue resistance, so that they less stimulate the surrounding tissues; (3) the combination of cable wires and pedicle screw fixation can further strengthen the fixation effect and promote patients' joint activities after surgery as soon as possible. ${ }^{[9-11]}$

This method has an excellent clinical therapeutic effect on the treatment of adult multi-segment lumbar spondylolysis, and it has a series of advantages such as small surgical trauma, fast postoperative recovery and so on. This technique can also restore the stability of spinal segments and relieve pains to a greater degree.

\section{CONFlicts OF InTEREST Disclosure}

The authors declare they have no conflicts of interest. 


\section{REFERENCES}

[1] Dong M, Ming-kun Y, Xu Z, et al. Intervertebral fusion cage combined with pedicle screw systems for the treatment of lumbar isthmic spondylolisthesis: evaluation of the intervertebral space height. Journal of Clinical Rehabilitative Tissue Engineering Research. 2014; 44: 7122-7126.

[2] Da H, WeiT, Qiang Y, et al. Computer Assisted Minimal Invasive Spine Surgery with translaminar lag screw fixation for simple spondylolysis in young patients. Chinese Journal of Orthopaedic Trauma. 2014; 16(3): 204-207.

[3] Zhi-bin Z, Jian-zhong H. Progress of surgical treatment in adult lumbar spondylolisthesis. China Modern Medicine. 2015; 22(20): 19-22.

[4] Dong F, Wan-zong W, Hai-yan S, et al. The surgical treatment of lumbar spondylolysis with a pedicle screw-lamina hook plus isthmic bone grafting. The Journal of Cervicodynia and Lumbodynia. 2013; 5: 391-392.

[5] Jian-lan W, Yi-liang P. Analysis on therapeutic effect of lamina hook combined with bone grafting on 12 cases of lumbar spondylolysis. Chongqing Medicine. 2012; 41(13): 1323-1324.

[6] Kobayashi A, Kobayashi T, Kato K, et al. Diagnosis of radiographically occult lumbar spondylolysis in young athletes by mag- netic resonance imaging. Am J Sports Med. 2013; 41(1): 169-176. PMid:23136176. https ://doi .org/10.1177/03635465124649 46

[7] Sairyo K, Sakai T, Yasui N, et al. Conservative treatment for pediatric lumbar spondylolysis to achieve bone healing using a hard brace: What type and how long? Clinical article. J Neurosurg Spine. 2012; 16(6): 610-614. PMid:22519929. https://doi.org/10.3171/20 12. 2. SPINE10914

[8] Bo W, Xi-jin F, Wei C, et al. Clinical application of modified Weinstin technique used as pedicle screw placement for lumbar spondylolisthesis. Orthopaedics. 2015; 6(2): 66-69.

[9] Ping W, Hao W, Chun-lei W, et al. Effect of TESSYS on VAS and ODI in senile patients with LDH and LSS. China Medical Equipment. 2018; 15(12): 109-113.

[10] Zheng W, Tiansheng S. Advance in the treatment of lumbar spondylolysis. Chinese Journal of Tissue Engineering Research. 2017; 27 : 4423-4428.

[11] Hong-da X, Jia-nan L, Hong-da L, et al. Vertebral three-dimensional motion characteristics of adjacent segments in patients with isthmic spondylolisthesis in vivo. Chinese Journal of Reparative and Reconstructive Surgery. 2018; 32(12): 1560-1566. 